\title{
Polarization diversity scheme for BOTDA sensors based on a double orthogonal pump interaction
}

\author{
Javier Urricelqui, Felipe López-Fernandino, Mikel Sagues, Alayn Loayssa
}

\begin{abstract}
We introduce a Brillouin optical time domain analysis (BOTDA) sensor deploying a novel polarization diversity technique based on the use of two orthogonal pump pulses which simultaneously interact with a phase-modulated probe wave. The orthogonallity of the two pump pulses guarantees that two complementary Brillouin interactions take place at each position of the fiber, so that polarization independent measurements are performed throughout the fiber even if no averaging is applied. This feature can be exploited in dynamic distributed BOTDA sensors to reduce the measurement time, as no extra averaging is required to compensate the polarization dependence of Brillouin interaction. Proof-of-concept experiments demonstrate the feasibility of the technique by employing a completely passive scheme to generate the orthogonal pump pulses. Furthermore, the technique is stable and easy to implement, making it a perfect candidate for practical sensor implementations.
\end{abstract}

Index Terms-Brillouin distributed sensors, Brillouin optical time domain analysis, Heterodyne, Optical fiber sensors, Stimulated Brillouin scattering.

\section{INTRODUCTION}

$\mathrm{D}$ STRIBUTED fiber sensors are very attractive for a wide set of applications, including structural health monitoring, where an increasing number of them require of the capability to perform dynamic temperature or strain measurements [1][2][3]. In this context, Brillouin optical time domain analysis (BOTDA) sensors provide high precision distributed measurements of the strain profile in extremely large structures. Nevertheless, measurement time in BOTDA sensors is typically of the order of a few minutes, mainly due to the need for scanning the Brillouin spectrum, in order to obtain the Brillouin frequency shift (BFS) of the fiber. As a result, BOTDA sensors have been traditionally limited to static measurements.

However, dynamic variations of temperature or strain can be measured by modifying the sensor technique. This can be

Manuscript received September, 13, 2014. The authors wish to acknowledge the financial support from the Universidad Pública de Navarra and Spanish Ministerio de Ciencia e Innovación through the project TEC2013-47264-C2-2-R.

Javier Urricelqui, Felipe López-Fernandino, Mikel Sagues and Alayn Loayssa are with Deparment of Electrical and Electronic Engineering, Universidad Pública de Navarra, Campus Arrosadía S/N, 31006, Pamplona, Navarre, Spain (e-mail: mikel.sagues@ unavarra.es) made by tuning the probe wave to the skirt of the Brillouin gain spectrum so that variations in BFS are translated to changes in the amplitude of the detected probe wave [4][5][6]. A key challenge faced by these dynamic BOTDA sensors is to reduce the extra time added to the measurement due to the need to compensate for the polarization dependence of Brillouin interaction. This polarization dependence is usually overcome by performing a time-domain polarization scrambling of the probe wave or of the pump pulses. However, this forces a higher averaging in the measurement, as a large number of states of polarization (SOP) must be averaged in order to obtain a polarization independent measurement. Another possibility is to use a polarization switch, so that two orthogonal SOPs are sequentially launched into the fiber [7]. However, this adds additional complexity to the system and the measurement time is limited by the switching time. A third approach is to implement a passive polarization scrambler using an unbalanced Mach-Zehnder interferometer [8]. This provides an excellent polarization scrambling with no time penalty in the measurement, but it's limited to sensor setups deploying single wavelength $\mathrm{CW}$ probe signals.

Recently, we proposed a dynamic BOTDA sensor based on a phase-modulated probe wave and self-heterodyne detection [9]. This technique enhances the SNR in relation to the conventional direct detection scheme and is able of performing measurements largely immune to attenuation in the optical fiber [10]. In this paper, we propose an improved setup based on the use of a double orthogonal pulsed pump, which retains the advantages of the previous technique and adds a new one: the capability to perform polarization independent measurements [11].

\section{FUNDAMENTALS OF THE POLARIZATION DIVERSITY TECHNIQUE}

Stimulated Brillouin scattering (SBS) process has its origin in the interference between two optical waves which generates a traveling longitudinal acoustic wave. This acoustic wave, in turn, couples the optical waves to each other. As the process is coherent in the optical domain, its efficiency is dependent on the relative states of polarization of the two waves involved in the process.

This polarization dependence of SBS interaction can be mathematically modeled by the real factor $\eta$ which determines the mixing efficiency of the counterpropagating signals [12]. 


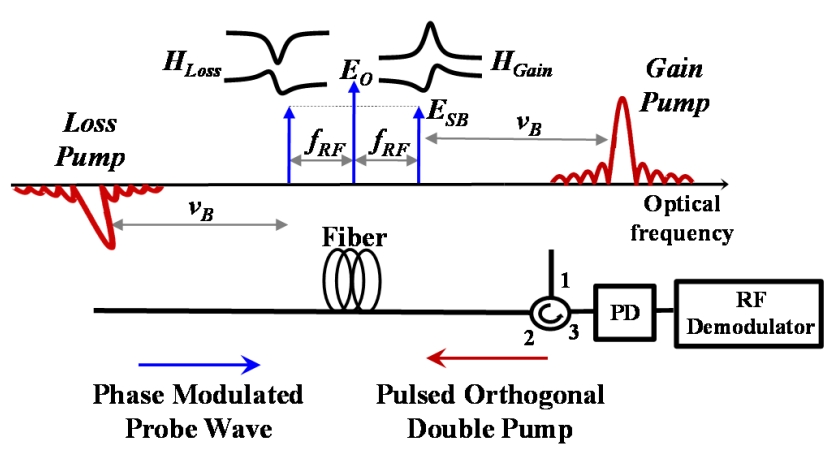

Fig. 1. Schematic representation of the fundamentals of the double orthogonal-pump SBS interaction, where each of the two orthogonal pump pulses (red) interacts with a different modulation sideband of the phase-modulated probe wave (blue).

In general, this mixing efficiency factor $\eta(0 \leq \eta \leq 1)$ can be described as a function of the SOP of probe and pump signals as:

$\eta=\frac{1}{2}\left(1+s_{1 p} s_{1 s}+s_{2 p} s_{2 s}-s_{3 p} s_{3 s}\right)$

where $\hat{\mathrm{S}}_{\mathrm{s}}=\left(\mathrm{s}_{1 \mathrm{~s}}, \mathrm{~s}_{2 \mathrm{~s}}, \mathrm{~s}_{3 \mathrm{~s}}\right)$ and $\hat{\mathrm{S}}_{\mathrm{p}}=\left(\mathrm{s}_{1 \mathrm{p}}, \mathrm{s}_{2 \mathrm{p}}, \mathrm{s}_{3 \mathrm{p}}\right)$ are the normalized Stokes vectors representing the SOP of the counterpropagating probe and pump signals, respectively, at a given position of the sensing fiber. Therefore, for a differential length of fiber, SBS interaction between pump and probe waves is optimal $(\eta=1)$ when the orientation, the ellipticity and the sense of rotation of their polarization ellipses are the same when seen from one direction. Conversely, no SBS interaction takes place $(\eta=0)$ when the polarization ellipses of pump and Stokes waves are similar to the first case, but with orthogonal orientation (their long axes are orthogonal to each other) [12][13].

In the most usual scenario for distributed fiber sensors, the measurement is performed over standard single-mode fiber, where the SOP of the counterpropagating waves will vary randomly and dynamically. Therefore, it is not possible to guarantee a proper alignment between them for every position of the fiber. As a result, the signal to noise ratio (SNR) of the measurement will be very low in some positions of the fiber, being zero for those where there is no Brillouin interaction. As a consequence, in Brillouin-based sensors it is necessary to apply some kind of polarization averaging or polarization diversity technique.

If we now consider the case where the state of polarization of the pump wave is orthogonal to the previous one $\left(\hat{\mathrm{S}}_{\mathrm{p}}\right)$, its normalized Stokes vector will be given by $\hat{\mathrm{S}}_{\mathrm{p}} \perp=\left(-\mathrm{s}_{1 \mathrm{p}},-\mathrm{s}_{2 \mathrm{p}},-\mathrm{S}_{3 \mathrm{p}}\right)$ and the mixing efficiency will yield:

$\eta_{\perp}=\frac{1}{2}\left(1-s_{1 p} s_{1 s}-s_{2 p} s_{2 s}+s_{3 p} s_{3 s}\right)$

Therefore, by simply adding $\eta$ and $\eta \perp$ in (1) and (2), respectively, it is found that both interactions will be complementary:

$\eta+\eta_{\perp}=1$
Furthermore, due to the unitary nature of the fiber, the two orthogonal polarization states entering the fiber will keep their relative orthogonallity, even though the states themselves are continuously changing along the fiber [13]. As a consequence, the complementarity of the two Brillouin interactions will be guaranteed throughout the whole length of fiber, even for relatively long fibers, where the orthogonalization error induced by polarization dependent loss is still negligible [14].

In our technique, we take advantage of this property by generating two simultaneous and complementary Brillouin interactions along the sensing fiber. Fig. 1 schematically depicts the fundamentals of the proposed system. A single tone phase-modulated probe wave is injected in one end of the optical fiber under test, while two orthogonal pump pulses are introduced at the other end. Each sideband of the phase-modulated probe wave interacts with its respective pump pulse via stimulated Brillouin scattering. The resulting phase-modulated probe wave affected by both quadrature-polarization Brillouin interactions is directed to the receiver using a circulator. Finally, the detected RF electrical signal is demodulated in a synchronous demodulator so that both the amplitude and the phase-shift of the detected RF signal can be recovered [10].

As schematically shown in Fig. 1, the wavelengths of the pump pulses are adjusted so that each of the two orthogonal pumps interacts with one of the modulation sidebands of the phase-modulated probe wave. The aim of this double interaction is to produce a Brillouin gain over one of the sidebands, while a Brillouin loss is generated over the second one. Notice that this two optical interactions of opposite sign will not cancel each other once the phase-modulated probe wave is detected, due to the fact that the two sidebands of the modulation have a phase-shift of $\pi$ radians [10].

If the modulation frequency of the probe wave is much higher than the Brillouin bandwidth, we can consider that each SBS interaction only affects one sideband of the modulation, leaving the other sideband and the carrier unaffected. Then, the optical field at the input of the photodetector coming from the interaction of pump and probe at a particular location in the fiber, $\mathrm{z}$, is given by the following expression:

$$
\begin{aligned}
E(t, z) & =E_{0} \exp \left(j 2 \pi v_{0} t\right) \\
& +E_{S B} \exp \left(j 2 \pi\left(v_{0}+f_{R F}\right) t\right) H_{S B S, \text { Gain }}\left(v_{0}+f_{R F}, z\right) \\
& -E_{S B} \exp \left(j 2 \pi\left(v_{0}-f_{R F}\right) t\right) H_{S B S, \text { Loss }}\left(v_{0}-f_{R F}, z\right)
\end{aligned}
$$

where $E_{0}$ and $E_{S B}$ are the amplitudes of the optical fields of the carrier and first sidebands of the phase-modulated probe wave (higher-order sidebands were neglected, assuming a small modulation index), $v_{0}$ is the optical frequency of the carrier, $f_{R F}$ is the modulation frequency and $H_{\text {Gain }}$ and $H_{\text {Loss }}$ are, respectively, the complex Brillouin gain and loss spectra at position z. In case of employing pump pulses longer than the acoustic lifetime, these spectra have a Lorentzian shape given by: 


$$
\begin{aligned}
H_{S B S}(\Delta v, z) & =\exp \left(\eta \frac{g_{B} \Delta v_{B}}{\Delta v_{B}+2 j \Delta v}\right) \\
& =\exp \left(\frac{\eta g_{B} \Delta v_{B}^{2}}{\Delta v_{B}^{2}+4 \Delta v^{2}}\right) \exp \left(-\frac{j 2 \eta g_{B} \Delta v_{B} \Delta v}{\Delta v_{B}+2 j \Delta v}\right)^{(5)} \\
& =\exp \left(\eta G_{S B S}\right) \exp \left(j \eta \varphi_{S B S}\right) \\
& \approx 1+\eta\left(G_{S B S}+j \varphi_{S B S}\right)
\end{aligned}
$$

where, $\mathrm{g}_{\mathrm{B}}$ is the Brillouin gain $\left(\mathrm{g}_{\mathrm{B}, \mathrm{G}}=\mathrm{g}_{\mathrm{B}}\right)$ or loss $\left(\mathrm{g}_{\mathrm{B}, \mathrm{L}}=-\mathrm{g}_{\mathrm{B}}\right)$ depending on the described SBS interaction, $\Delta v_{B}$ is the Brillouin linewidth, $\Delta v=v-v_{P}+v_{B}(z)$ is the detuning of the interaction frequency from the center of the Brillouin spectrum, $v_{P}$ is the optical frequency of the pump wave and $v_{B}$ is the Brillouin frequency shift at position $z$. The approximation in the last term of (5) is obtained assuming a small gain (or loss), which is the case of BOTDA sensors. Note also that, $\mathrm{G}_{\mathrm{SBS}}$ and $\varphi_{\mathrm{SBS}}$ represent, respectively, the Brillouin gain (or loss) factor and the Brillouin phase-shifts of each interaction shown in Fig.1. Furthermore, the symmetry of both Brillouin interactions is so, that the phase-shift induced over both sidebands will be equal $\left(\varphi_{S B S}=\varphi_{G}=\varphi_{L}\right)$, and the gain factor will be of identical magnitude but opposite sign $\left(G_{S B S}=G_{G}=-G_{L}\right)$. In that case, the detected optical power signal at the demodulation frequency $f_{R F}$ can be expressed as:

$$
\begin{aligned}
\left.P(t)\right|_{f_{R F}} \approx\left[\left(1+\eta_{G}\left(G_{G}+j \varphi_{G}\right)\right)-\left(1+\eta_{L}\left(G_{L}-j \varphi_{L}\right)\right)\right] \\
\cdot 2 E_{0} E_{S B} \cos \left(2 \pi f_{R F} t\right) \\
=2 E_{0} E_{S B}\left(\eta_{G}+\eta_{L}\right)\left(G_{S B S}+j \varphi_{S B S}\right) \cos \left(2 \pi f_{R F} t\right)
\end{aligned}
$$

If we now consider that both sidebands of the phase-modulated probe wave have the same SOP, then (3) can be applied so that $\eta_{G}$ and $\eta_{L}$ can be removed from the equation. Therefore, the technique removes the need for polarization scrambling, as the detected signal for any given position of the fiber will be independent of the SOP of the probe wave. As a consequence, no averaging is required to eliminate the polarization noise.

Substituting (5) in (6), we finally obtain the expression of the detected RF signal using the presented technique:

$$
\begin{aligned}
\left.P(t)\right|_{f_{R F}} & \approx \frac{2 E_{0} E_{S B} g_{B} \Delta v_{B}}{\sqrt{\Delta v_{B}^{2}+(2 \Delta v)^{2}}} \\
& \cdot \cos \left(2 \pi f_{R F} t-\arctan \left(2 \frac{\Delta v}{\Delta v_{B}}\right)\right)
\end{aligned}
$$

Equation (7) shows that the detected RF phase-shift is independent of the particular Brillouin gain peak experienced by the probe wave and of the received optical power. This is a key feature for dynamic measurements, as the sensor will not be susceptible to errors caused by changes in pump power or attenuation in the fiber, which are highly probable in a structure with dynamic deformations [10]. Therefore, the same technique described in [10] can be deployed to perform dynamic measurements, but with the additional advantage of having polarization independent BOTDA traces.

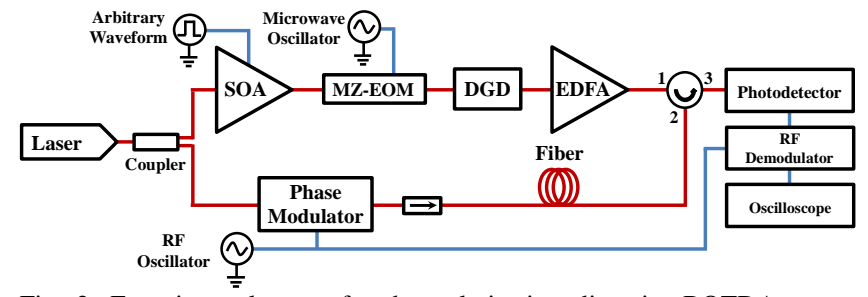

Fig. 2. Experimental setup for the polarization diversity BOTDA sensor based on a phase-modulated probe wave, a double orthogonal pump and RF demodulation.

\section{EXPERIMENTAL SETUP AND MEASUREMENTS}

The experimental setup shown in Fig. 2 was assembled in order to demonstrate the system. The output of a $1550.5 \mathrm{~nm}$ laser source is divided in two optical branches with an optical coupler. In the upper branch, the optical beam is pulsed using a semiconductor optical amplifier (SOA). This pulsed beam is directed to a Mach-Zehnder electrooptic modulator (MZ-EOM) driven by a microwave signal of $9.6 \mathrm{GHz}$ and biased in minimum transmission, so that two pulsed sidebands are generated, while the optical carrier is suppressed.

In order to orthogonalize the SOP of the two pulsed sidebands of the optical double sideband with suppressed carrier (ODSB-SC) modulation, an all-optical, passive and simple technique is implemented. This is performed by the use of a differential group delay (DGD) module, which provides a group delay between two linear orthogonally polarized waves. The technique is schematically described in Fig. 3. The linearly polarized light coming from the MZ-EOM is launched into the birefringent material at $45^{\circ}$ or $135^{\circ}$ with the axis of the DGD module. This feature can be provided by the manufacturer, so that the connection of the DGD module to the output of a MZ-EOM with polarization maintaining fiber is straightforward, requiring no additional polarization control. After going through the DGD module, the differential phase-shift suffered by the light travelling through the two principal axis of the DGD depends on its wavelength $(\lambda)$ :

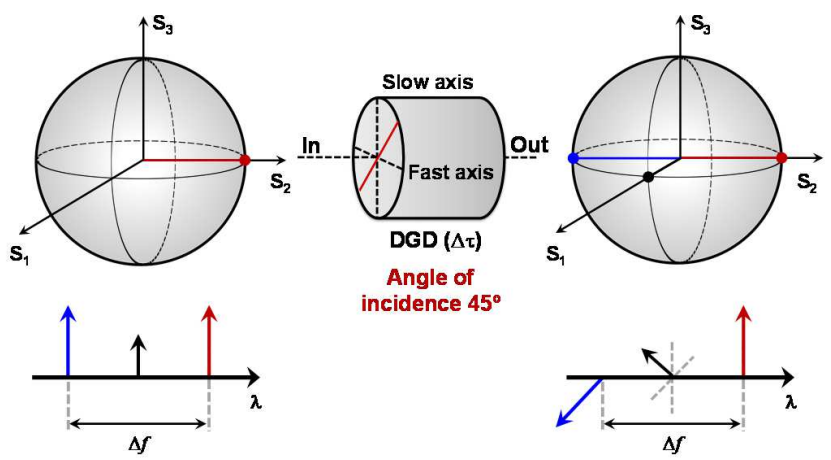

Fig. 3. Schematic representation of the procedure deployed to orthogonalize the sidebands of the optical modulation at the output of the MZ-EOM. The optical carrier and the modulation sidebands have the same SOP when they impinge the DGD module, but the differential delay suffered by each of them makes the two modulation sidebands orthogonal. 


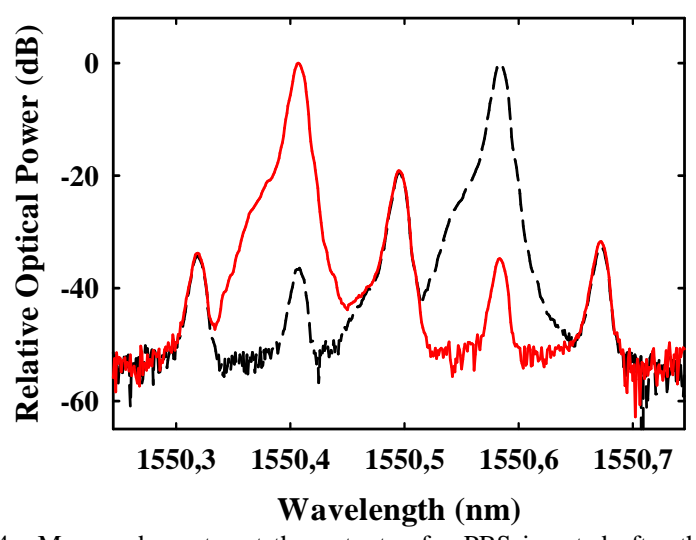

Fig. 4. Measured spectra at the outputs of a PBS inserted after the DGD module, when the state of polarization of one of the sidebands of the modulation is aligned with the principal axis of the PBS (red solid line and black dashed line).

$\theta(\lambda)=\frac{2 \pi L\left(n_{e}-n_{o}\right)}{\lambda}$

where $L$ is the length of the DGD module, and $n_{e}$ and $n_{o}$ are, respectively, the extraordinary and ordinary refraction indexes of the DGD module. Therefore, depending on the wavelength of the incident beam, the birefringent material provides different polarization states at its output [15]. Notice that if the wavelengths of two waves are tuned in such a way that the difference between the relative phase-shifts suffered by them is made equal to $\pi$, their SOP will be orthogonal at the output of the DGD.

$\Delta \theta=2 \pi \cdot \Delta f \cdot \Delta \tau=\pi$

where $\Delta \tau$ is the differential group delay introduced by birefringence and $\Delta \mathrm{f}$ the frequency difference between both waves. As a result, in order to obtain linear orthogonal polarizations between both sidebands of the ODSB-SC modulation, the $\Delta \tau$ needed is $1 / 4 f_{\mu}$, where $f_{\mu}$ is the frequency of the microwave signal driving the MZ-EOM.

In this way, the two pump waves are made orthogonal by simply inserting in the setup an inexpensive, passive and easy to deploy component. In our experiment, a DGD module with $\Delta \tau=26 \mathrm{ps}$ was used, so that the two pulsed sidebands of the modulation, separated by $19.2 \mathrm{GHz}$, were made orthogonal.

In order to verify the orthogonallity of the generated pump pulses, a polarization controller and a polarization beam splitter (PBS) where inserted after the DGD module in the experimental setup. Fig. 4 shows the spectra at the two outputs of the PBS, measured in an optical spectrum analyzer (OSA), when the SOP of one of the sidebands at the input of the PBS is aligned with one of its principal axis. A sideband suppression of more than $30 \mathrm{~dB}$ is observed, showing an excellent orthogonallity between both sidebands. The modulation was not pulsed during this measurement so that the spectrum could be properly acquired in the OSA.

Once the double orthogonal pump is generated, this is amplified by an erbium doped fiber amplifier (EDFA), and the

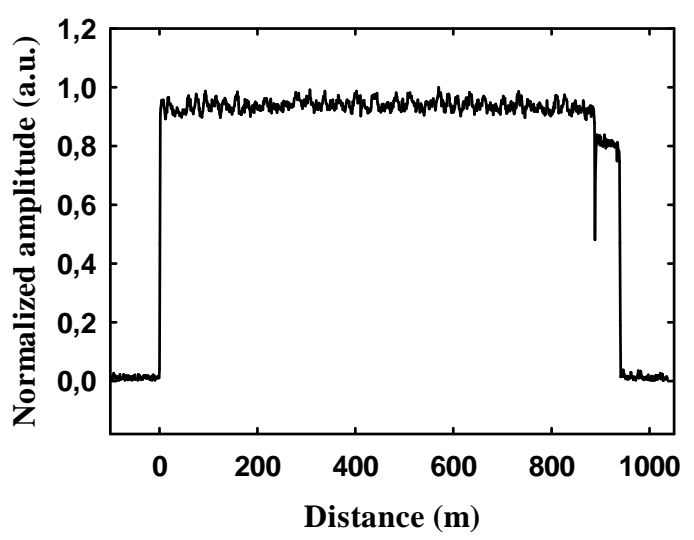

(a)

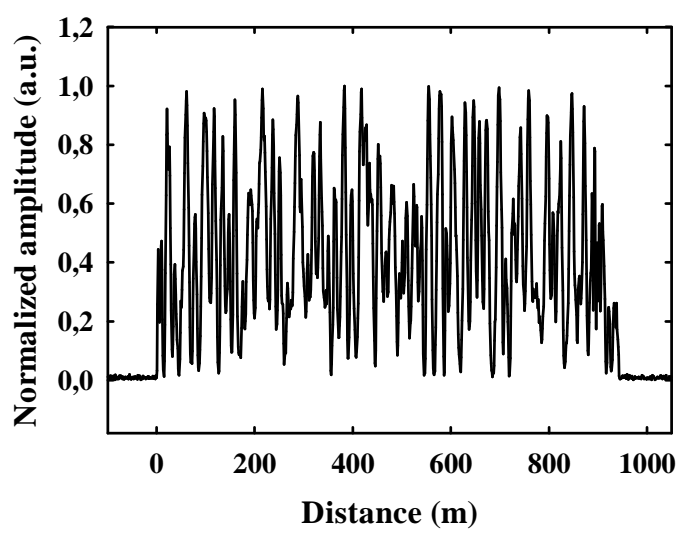

(b)

Fig. 5. Measured BOTDA trace (a) when the polarization diversity technique is implemented and (b) when the DGD module is removed from the setup.

resulting pump pulses are directed via a circulator to a 930m-long fiber.

In the lower branch of the setup in Fig. 2, the probe wave is generated with an electro-optic phase modulator driven by a $1.3 \mathrm{GHz} \mathrm{RF}$ signal. The modulation frequency is chosen so that the upper sideband of this modulation interacts via SBS with the lower sideband of the ODSB-SC modulated pump pulse, while the lower sideband of the phase modulation is affected by the higher frequency pulsed pump. After interacting with the orthogonal pump pulses via SBS, the probe signal is directed to a receiver and the resultant RF signal is demodulated [10]. Finally, the BOTDA signal is captured in a digital oscilloscope.

The BOTDA trace captured in the oscilloscope is depicted in Fig. 5 (a), showing negligible polarization dependence, as compared with a conventional BOTDA trace (Fig.5 (b)). The trace in Fig.5 (b) was obtained by removing the DGD module from the setup. Both traces have been acquired using a 128 averaging rate, but deploying no further technique to compensate the polarization dependence of SBS interaction.

In order to perform dynamic measurements, a 1-meter section at the end of the 930-m fiber was affixed by epoxy resin onto the surface of a 1-m cantilever beam. The cantilever beam was made to vibrate so that dynamic distributed measurements of the induced strain along the fiber could be performed. The pulse duration was set to $10 \mathrm{~ns}$ to obtain 1-m spatial resolution. Moreover, a BOTDA trace was captured 


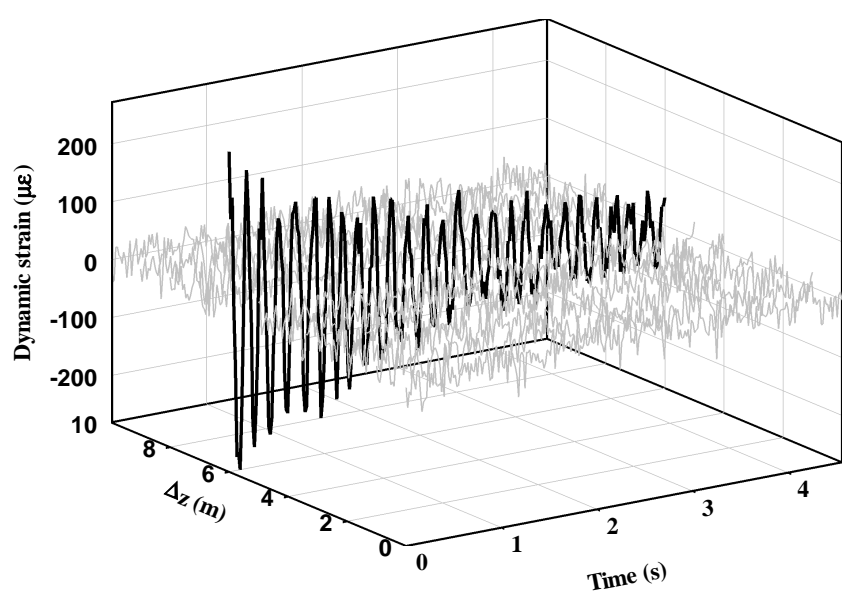

Fig. 6. Fast-acquisition polarization-compensated measurement of the strain induced at the last locations of the fiber (grey traces), where the cantilever beam (black solid line) is made to vibrate.

every $12 \mu \mathrm{s}$ and 128 averages were used, achieving a $651 \mathrm{~Hz}$ measurement rate. Fig. 6 shows the measured strain in the final locations of the fiber when the cantilever beam is made to vibrate. The distributed measurement is also demonstrated, as only the section of the fiber attached to the cantilever beam is suffering strain, while the adjacent sections of fiber remain steady. The estimated uncertainty was $2 \mathrm{MHz}$ for a $145 \mathrm{MHz}$ $(2900 \mu \varepsilon)$ measurement range measured using the guidelines given in [10]. These parameters can be enhanced by increasing the SNR parameter of the sensor or reducing its phase noise [16]. For this particular case, the performance is similar to the state or the art in dynamic sensors in terms of measurement rate, spatial resolution or length of fiber; although the precision, which is limited by the instrumentation deployed, is slightly lower than in some contributions [6] [17]. However, our technique presents some key advantages comparing to other dynamic BOTDA setups. Specifically, the measurement range is larger than in the conventional slope-assisted BOTDA technique due to the deployment of the phase spectrum to translate variations of the detected phase into changes of the BFS of the fiber [10]. Besides, the use of the RF phase-shift spectrum also provides immunity to pump or probe power variations, which are one of the main error sources in amplitude-based slope-assisted BOTDA sensors [10].

In practical applications, the static BFS distribution is usually non-uniform, as different types of fiber, or fibers suffering different strain or temperatures can be found through the sensing length of the sensor. In this case, conventional dynamic slope-assisted BOTDA sensors can't be deployed even if the dynamic strain changes are small, due to their limited measurement range. This limitation can be overcome by deploying pump or probe waves that vary their frequency dynamically in order to fit the Brillouin profile of the fiber [6]. Notice that our technique is compatible with the one introduced in [6] so that the advantages of both can be put together.

\section{CONCLUSIONS}

A dynamic Brillouin distributed sensor featuring a novel polarization diversity scheme based on a double orthogonal pump interaction has been proposed and demonstrated. The technique relies on the fact that, for any given location of the fiber, both Brillouin interactions remain complementary, so that their sum is always equal to a single Brillouin interaction with aligned SOP for probe and pump waves. Therefore, the technique removes the need for polarization scrambling or for any other polarization compensating technique, so that the measurement time can be reduced. A theoretical model has been developed and the capabilities of this novel scheme have been experimentally demonstrated, performing a fast dynamic distributed strain measurement in a cantilever beam with high measurement range and precision. Besides, the practical implementation of the double orthogonal pump, relies on the use of a DGD module, which is an inexpensive optical component that can be introduced in the setup without requiring any polarization control. This makes it very robust to environmental changes, so that it can be considered as a solution for commercial sensors.

\section{REFERENCES}

[1] X. Bao and L.Chen, "Recent progress in Brillouin scattering based fiber sensors," Sensors, vol. 11, no. 4, pp. 4152-4187, Apr. 2011.

[2] K. Y. Song and K. Hotate, "Distributed fiber strain sensor with 1-kHz sampling rate based on Brillouin optical correlation domain analysis," IEEE Photon. Technol. Lett., vol. 19, no. 23, pp. 1928-1930, Dec. 2007.

[3] K. Y. Song, M. Kishi, Z. He and K. Hotate, "High-repetition-rate distributed Brillouin sensor based on optical correlation-domain analysis with differential frequency modulation," Opt. Lett., vol. 36, no. 11, pp. 2062-2064, June 2011.

[4] R. Bernini, A. Minardo and L. Zeni, "Dynamic strain measurement in optical fibers by stimulated Brillouin scattering," Opt. Lett., vol. 34, no. 17, pp. 2613-2615, Sept. 2009.

[5] Q. Cui, S. Pamukcu, W. Xiao and M. Pervizpour, "Truly distributed fiber vibration sensor using pulse base BOTDA with wide dynamic range," IEEE Photon. Technol. Lett., vol. 23, no. 24, pp. 1887-1889, Dec. 2011.

[6] Y. Peled, A. Motil and M. Tur, "Fast Brillouin optical time domain analysis for dynamic sensing," Opt. Express, vol. 20, no. 8, pp. 85848591, Apr. 2012.

[7] K. Hotate, K. Abe and K. Y. Song, "Suppression of signal fluctuation in Brillouin optical correlation domain analysis system using polarization diversity scheme," IEEE Photon. Technol. Lett., vol. 18, no. 24, pp. 2653-2655, Dec. 2006

[8] S. Díaz, S. M. Foaleng, M. López-Amo and L. Thévenaz, "High performance Brillouin distributed fibre sensor," Proc. of SPIE, vol. 6619, July 2007.

[9] A. Zornoza, M. Sagues and A. Loayssa, "Self-heterodyne detection for SNR improvement and distributed phase-shift measurements in BOTDA,” J. Lightwave Technol., vol. 30, no. 8, pp. 1066-1072, Apr. 2012.

[10] J. Urricelqui, A. Zornoza, M. Sagues and A. Loayssa, "Dynamic BOTDA measurements based on Brillouin phase-shift and RF demodulation," Opt. Express, vol. 20, no. 24, pp. 26942-26949, Nov. 2012.

[11] J. Urricelqui, F. López-Fernandino, M. Sagues and A. Loayssa, "Polarization diversity for Brillouin distributed fiber sensors based on a double orthogonal pump," Proc. of SPIE, vol. 9157, June 2014.

[12] M. O. van Deventer and A. J. Boot, "Polarization properties of stimulated Brillouin scattering in single-mode fibers," J. Lightwave Technol., vol. 12, no. 4, pp. 585-590, Apr. 1994.

[13] A. Zadok, E. Zilka and A. Eyal, "Vector analysis of stimulated Brillouin scattering amplification in standard single-mode fibers," Opt. Express, vol. 16, no. 26, pp. 21692-21707, Dec. 2008.

[14] G. D. VanWiggeren and R. Roy, "Transmission of linearly polarized light through a single-mode fiber with random fluctuations of birefringence,” Appl. Opt. vol. 38, no. 18, pp. 3888-3892, June 1999.

[15] J. P. Gordon and H. Kogelnik, "PMD fundamentals: Polarization mode dispersion in optical fibers," P. Natl. Acad. Sci. USA 97, vol. 97, no. 9, pp. 4541-4550, Feb. 2000. 
[16] A.B. Carlson, P.B. Crilly, and J.C: Rutledge, "Noise in analog modulations systems," in Communications Systems: An Introduction to Signals and Noise in Electrical Communication, 4th ed., New York, McGraw-Hill, 2002, ch. 10 pp. 398-429.

[17] Y. Peled A. Motil, I. Kressel, and M. Tur, "Monitoring the propagation of mechanical waves using an optical fiber distributed and dynamic strain sensor based on BOTDA," Opt. Express, vol. 21, no. 9, pp. 1069710705, May 2013 
(C) 2015 IEEE. Personal use of this material is permitted. Permission from IEEE must be obtained for all other uses, in any current or future media, including reprinting/republishing this material for advertising or promotional purposes, creating new collective works, for resale or redistribution to servers or lists, or reuse of any copyrighted component of this work in other works. 